\title{
Enstrophy amplification events in three-dimensional turbulence
}

Jörg Schumacher, Maik Boltes, Herwig Zilken, Marc-André Hermanns, Bruno Eckhardt, and Charles R. Doering

Citation: Chaos 18, 041103 (2008); doi: 10.1063/1.2997336

View online: https://doi.org/10.1063/1.2997336

View Table of Contents: http://aip.scitation.org/toc/cha/18/4

Published by the American Institute of Physics

\section{Smarter Search $\mathbf{Q}$ with the redesigned Physics Today Buyer's Guide}

Find the tools you're looking for today! 


\section{Enstrophy amplification events in three-dimensional turbulence}

Jörg Schumacher, ${ }^{1}$ Maik Boltes, ${ }^{2}$ Herwig Zilken, ${ }^{2}$ Marc-André Hermanns, ${ }^{2}$ Bruno Eckhardt, ${ }^{3}$ and Charles R. Doering ${ }^{4}$

${ }^{1}$ Technische Universität Ilmenau, D-98684 Ilmenau, Germany ${ }^{2} J u ̈ l i c h$ Supercomputing Centre, D-52425 Jülich, Germany ${ }^{3}$ Philipps-Universität Marburg, D-35032 Marburg, Germany

${ }^{4}$ The University of Michigan Ann Arbor, Ann Arbor, Michigan 48109-1043, USA

(Received 17 September 2008;

published online 30 December 2008)

[DOI: $10.1063 / 1.2997336]$

High-amplitude vorticity regions in turbulence are mostly found in the form of tube or sheetlike structures. The upper panel shows the isosurfaces of the vorticity magnitude obtained within a high-resolution pseudospectral simulation of homogeneous isotropic and forced turbulence on a grid of $2048^{3}$ points at a Taylor microscale Reynolds number of 107. An adequate resolution of the high vorticity requires a spectral resolution that is a factor 8 better than usual. ${ }^{1}$ In order to follow the temporal growth of the enstrophy-a bulk measure of the vorticity magnitude-we track velocity fields along Lagrangian trajectories in subvolumes with $51^{3}$ grid points. The middle panel shows one such subvolume together with isosurfaces of the vorticity magnitude at a level of ten times the root mean square vorticity value. ${ }^{2}$ One hundred of these subvolumes have been tracked simultaneously in the box turbulence.

The lower panel shows the enstrophy at the maximum of one such growth event. It displays two nested isolevels of the vorticity magnitude (red larger than gray). We observe two interacting vortex tube segments which are just about to form a strong shear layer in between. The highest-amplitude shear and vorticity events are found close in space and time to each other. The local temporal growth rate of the enstrophy can become as fast as the recently predicted upper bound, $d E / d t \sim E^{3}$, where $E$ is the enstrophy of the whole box. ${ }^{3}$

Supercomputations were conducted on 512 CPUs of the IBM p690 cluster JUMP at the Jülich Supercomputing Centre within the Deep Computing Initiative of the European DEISA consortium. Support by the Deutsche Forschungsgemeinschaft (DFG) and the U.S. National Science Foundation (NSF) is also gratefully acknowledged.

${ }^{1}$ J. Schumacher, K. R. Sreenivasan, and V. Yakhot, New J. Phys. 9, 89 (2007).

${ }^{2}$ J. Schumacher, B. Eckhardt, and C. R. Doering (in preparation).

${ }^{3}$ L. Lu and C. R. Doering, Indiana University Mathematics Journal (in press).
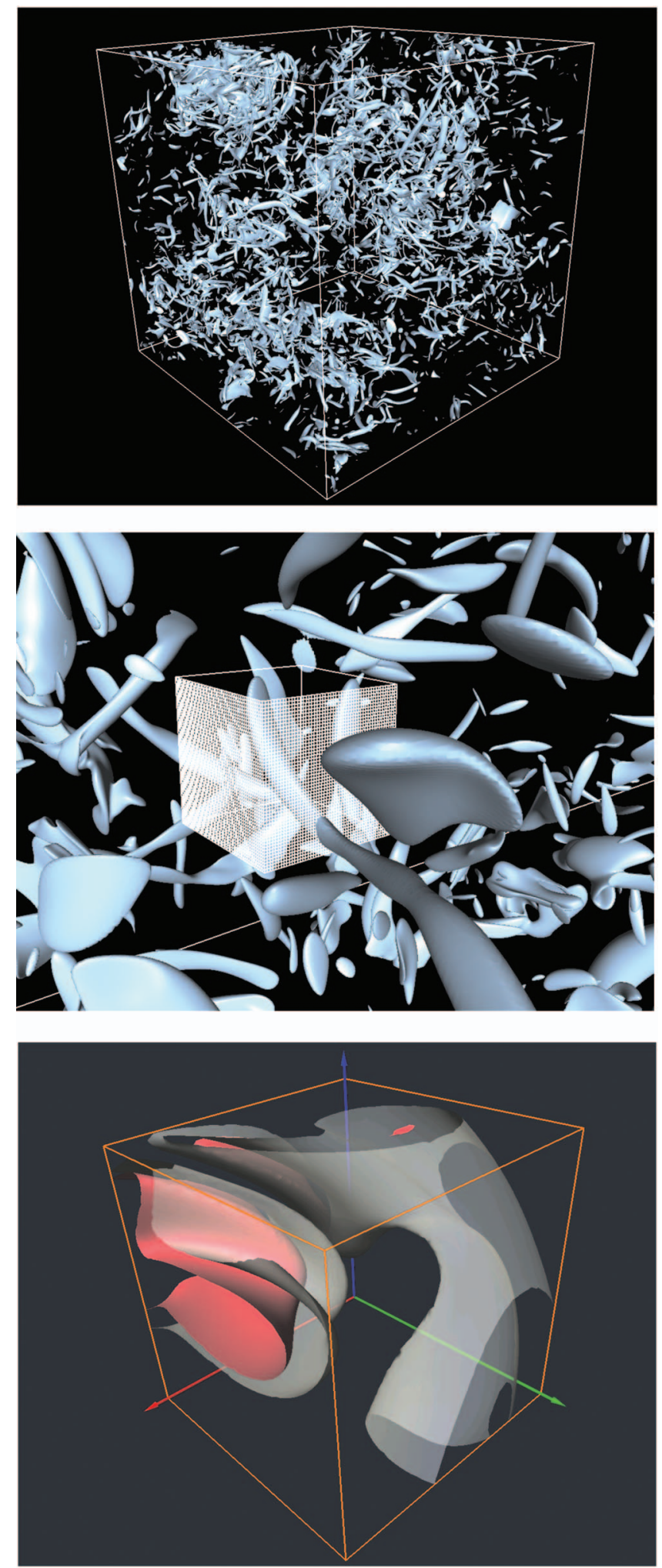

FIG. 1. (Color) (Enhanced online.) 